\title{
Changes in Neural Correlates of Outcome Feedback Processing During Implicit Learning
}

\author{
M. Rostami*,a ${ }^{\text {, S.M.H. Hosseini }}{ }^{\text {a,b,c }}$, M. Takahashi ${ }^{\text {a }}$, M. Sugiura ${ }^{c}$ and R. Kawashima ${ }^{\text {b,c }}$ \\ ${ }^{a}$ Dept. of Management Science and Technology, Graduate School of Engineering, Tohoku University, Sendai, Japan \\ ${ }^{b}$ Smart Aging International Research Center, Institute of Development, Aging and Cancer, Tohoku University, Sendai, \\ Japan \\ ${ }^{c}$ Dept. of Functional Brain Imaging, Institute of Development, Aging and Cancer, Tohoku University, Sendai, Japan
}

\begin{abstract}
In recent years, the neural substrates underlying outcome feedback processing have been investigated in several neuroimaging studies of feedback-based learning. However, what has been missed in these studies is that, the learning process itself also affects the way the feedback is being processed. In this study, we tried to investigate the changes in neural substrates underlying positive and negative feedback processing during goal-directed implicit learning using the Sugar Production Factory (SPF) task in conjunction with an event related functional magnetic resonance imaging. We found a significant learning-related decrease in activity of the right superior frontal gyrus (SFG) in response to positive feedback and a learning-related increase in activity of the precuneus in response to negative feedback. The results demonstrate the changing role of feedback during learning and suggest that learning-related changes in activity of the SFG and precuneus that have been previously reported in several implicit learning studies arise from changes in feedback processing after learning. In addition, the results suggest the important role of positive feedback in early stage and negative feedback in late stage of goal-directed implicit learning.
\end{abstract}

Keywords: Implicit learning, Positive and negative feedback, fMRI.

\section{INTRODUCTION}

Feedback processing is a crucial element of learning. Monitoring the outcome of our decisions is a prerequisite for the refinement of actions. We usually learn from both positive and negative outcomes of our actions, which include reinforcement of successful and avoidance of erroneous behavior, respectively [1]. Neural correlates of positive and negative feedback processing in human brain have been investigated in several neuroimaging studies. They reported activations in striatum, medial orbitofrontal cortex, medial and superior frontal cortex in response to positive feedback and activations in anterior cingulate, lateral orbitofrontal cortex, ventrolateral and dorsolateral prefrontal cortex, midbrain and insula in response to negative feedback processing [2-8].

In recent years, a number of neuroimaging studies of outcome feedback processing tried to identify brain regions which respond to feedback during the course of learning [911]. However, what has been missed in these studies is that, the learning process itself also affects the way we process the feedback. At the beginning of learning, subjects have no prior knowledge about the stimulus-response-outcome associations and therefore their decisions have no basis, while after learning, subjects make decisions based on a learned stimulus-response-outcome association. Therefore, feedback has a different role at the beginning of learning compared to

*Address correspondence to this author at the Dept. of Management Science \& Technology, Graduate School of Engineering, Tohoku University, 6-611-808, Aramaki-Aza-Aoba, Aoba-ku, Sendai, 980-8579, Japan; Tel: +8122-7953865; Fax: +81-22-7954847; E-mail: maria.rostami@gmail.com the feedback after learning which provides a true assessment of the task. Recently, Sailer and colleagues [12] investigated the changing role of feedback during learning a decisionmaking task in an fMRI study. They compared brain activities during an initial exploratory phase which was characterized by chance-level performance with those during proficiency phase in which subjects had learned the correct response strategy. However, they reported that in the proficiency phase, subjects had made correct choices, only. Therefore, the outcome feedback in the proficiency phase was completely predictable; implying that performance in the proficiency phase was top-down rather than feedbackdependent.

The goal of the present study is to investigate the changes in neural substrates underlying positive and negative feedback processing during exploratory phase and strategy acquisition phase in which subjects' performance is significantly improved by training but the feedback is still unpredictable while including more information. We investigated the changing role of feedback before and after training on a goal-directed implicit learning paradigm in which subjects learn associations between responses and the incentive value of outcomes while they make decisions to reach a goal and receive feedback on the outcome of their efforts along the way [13]. Unlike habit learning systems in which subjects learn associations between stimuli and responses without any link to the outcome that response engendered, in goaldirected learning subjects generate more outcome-dependent responses. Therefore, outcome feedback plays an important role in this type of learning. 
Sugar Production Factory (SPF) task, as a goal-directed implicit learning paradigm, in conjunction with an event related functional magnetic resonance imaging (fMRI) has been used [14]. The sugar production factory task is a wellknown task in psychological studies on implicit learning in which learning relies upon trial-by-trial feedback on decision outcomes [15-17]. In the task, subjects take on the role of the manager of a simple sugar production factory and are expected to learn implicitly from their previous experiences to achieve the goal of the task. The objective is to reach and maintain a specific level of sugar production (target) across trials by varying the number of workers employed. Each time the subject enters the number of workers employed (input), the sugar output is updated, and the subject then enters a new value for the number of workers. Thus, subjects have continuous feedback on the output variable, while they manipulate the input variable. However, the task is designed so that no unique output is associated with any one input.

The neural basis of learning the SPF task has been previously reported in our fMRI study [14]. Analyzing the learning process in the same experiment showed the specific role of the superior frontal gyrus (SFG) in early stage of learning which has been attributed to specific action-outcome associations or goal-directed nature of the SPF task $[18,19]$. The results of a previous lesion study on rats showed that this brain region is selectively involved in the acquisition of action-outcome associations in goal-directed learning [20]. Performing event-related analysis on positive and negative feedback events in the present study would help us clarify the probable role of the SFG in feedback processing during goal-directed learning.

Another brain region that showed significant learningrelated changes in activity in the same experiment was precuneus. Although activity in this brain region has been reported in previous implicit learning studies, it was either not discussed or merely mentioned as probable task-related activations [21, 22]. Eliassen et al. [23] showed that the precuneus exhibited greater activation for incorrectly than correctly performed trials in an associative learning task. They speculated that this activation could be associated with error processing. The results of the present study may also shed light on the probable role of the precuneus in feedback processing during implicit learning.

\section{MATERIALS AND METHODS}

\section{Subjects}

Twelve right-handed university students (all males; aged 19-27 years old; mean age 21 years) participated in this study. All subjects were healthy (no sign or history of medical or neurological diseases). We assessed their handedness using the Edinburgh Handedness Inventory [24]. Written informed consent was obtained from each subject in accordance with the guidelines approved by A Strategic Research and Education Center for an Integrated Approach to Language, Brain and Cognition, Tohoku University 21st Century Center of Excellence Program in Humanities and the 1975 Declaration of Helsinki. One subject was excluded from the analysis because of excessive head motion.

\section{Sugar Production Factory (SPF) Task}

In the SPF task, subjects were instructed to imagine that they were in charge of a hypothetical sugar production fac- tory. The goal was to reach and maintain a specific level of sugar production (target) across trials by manipulating the workforce. In each trial, the current number of workers, current production level, and target level were displayed (Fig. 1A). In this task, subjects had continuous feedback on the sugar production level, while they manipulated the workforce. Each time the subject entered the number of workers employed (input), the sugar output was updated. The size of the work force varied in 12 discrete steps, so that in any one trial, one could employ between 1 and 12 workers and 12 possible outputs existed ranging from 1000 to 12,000 tons. The lower and upper bounds were fixed at 1000 and 12,000 tons, respectively. Subjects could change the number of workers using three buttons, one button to increase it, one to decrease it, and the third to fix the desired number of workers. Subjects were asked to start to respond when the color of the worker block changed to blue. In each trial, the current workforce and production level were displayed.

Unbeknownst to the participants, the level of production was related to the work force by the equation

$$
\mathrm{S}=2 * \mathrm{~N}-\mathrm{S}_{\mathrm{l}},
$$

where $\mathrm{N}$ is the number of workers (1-12), $\mathrm{S}_{1}$ is the previous sugar output divided by $1000(1-12)$, and $S$ is the current sugar output. The computer adds 1,0 , or -1 on a random basis to $\mathrm{S}$ in each trial. The final $\mathrm{S}$ value is then converted to thousands of tons of sugar output. The complex nature of the equation is such that no unique output is associated with any one input. The resulting output depends on the previous output as well as the new work force figure entered by the subject.

In the SPF task, subjects reliably learn to improve their performance using outcome feedback. Subjects were told that if their response leads to achieving the target value (and +/- 1000), they are correct; Otherwise, they are wrong.

\section{Control Task}

In the control task, the target value was assigned to be zero. The subjects were instructed to check the sugar production value (1-12), enter the same number for workers, and then press the fixation button (Fig. 1B). The response cue in the control task was the same as in the SPF task. The presented numbers in the control task were designed so that the number of button presses was equal to that of the last task condition to control for motor output.

\section{Experimental Design}

The study consisted of two fMRI scanning sessions, with one training session in between. Before the first scan session, subjects were instructed to read the experimental instructions and to learn how to do the task in the MRI scanner. After preliminary adaptation to the manipulation of buttons, a first scan session in the MRI scanner was performed. After the first scan session, subjects were given $60 \mathrm{~min}$ outside the MRI scanner to practice the task until they achieved a $20 \%$ increase in performance. After this training period, a second scan session was performed in the MRI scanner.

The design of this experiment is shown in Fig. (1C). Experiments in the first scan session and second scan session consisted of nine runs. The runs themselves were made up of 15 trials of the SPF task and 15 trials of control task, sepa- 


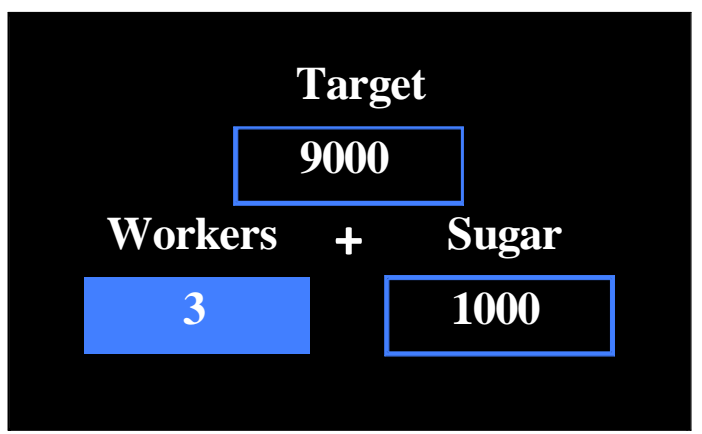

A

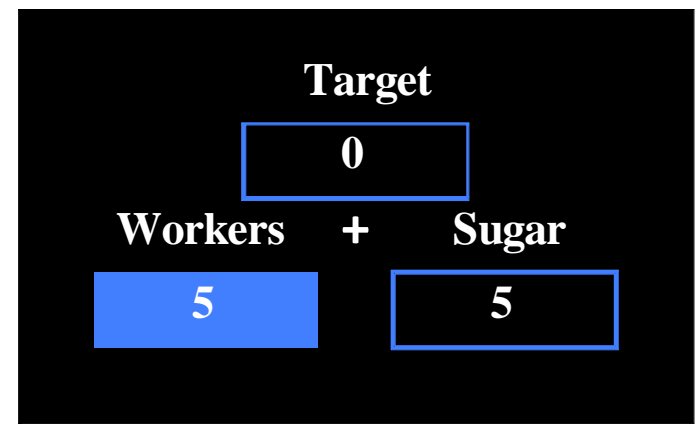

B

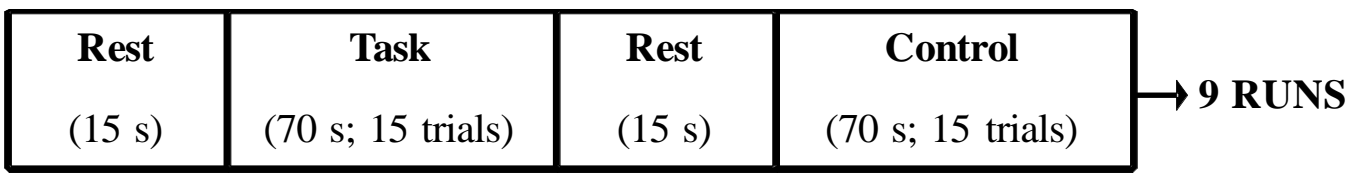

C

Fig. (1). (A) Schematic of the task. (B) Schematic of the control task. (C) Time course of the experiment; experiments in the first scan session and second scan session consisted of nine runs. The runs themselves were made up of 15 trials of the SPF task and 15 trials of control task, separated by $15 \mathrm{~s}$ of rest conditions.

rated by $15 \mathrm{~s}$ of rest conditions. The design of the task was self-paced. Thus, the inter-trial interval was varied among different trials for better estimation of the hemodynamic response.

\section{Imaging Procedure}

Slices $(\mathrm{n}=33$, slice thickness $=3 \mathrm{~mm}$, gap $=1 \mathrm{~mm})$ covering the whole brain were acquired by gradient-echo echoplaner (GE-EPI) magnetic resonance imaging (MRI) (repetition time $=3000 \mathrm{~ms}$, echo time $=50 \mathrm{~ms}$, flip angle $=90^{\circ}$, FOV $=192 \times 192 \mathrm{~mm}^{2}$, voxel size $=3 \times 3 \times 4 \mathrm{~mm}^{3}$, matrix $=$ $64 \times 64)$ on a $1.5 \mathrm{~T}$ Siemens Magnetom Symphony scanner (Siemens, Munich, Germany). A T1-weighted structural image was also acquired for each subject (192 slices, slice thickness $=1.0 \mathrm{~mm}$, slice gap $=1.0 \mathrm{~mm}, \mathrm{TR}=2200 \mathrm{~ms}$, TE $=3.93 \mathrm{~ms}$, FOV = $256 \mathrm{~mm}$ ).

\section{Data Analysis}

Image processing and statistical analyses of fMRI data were carried out using the statistical parametric mapping (SPM5) software [25, 26]. The three initial scans for each subject were dummy scans to equilibrate the state of magnetization and were discarded from the analysis. Differences in acquisition timing across slices in each scan were adjusted and the effects of head motion across the scans were corrected by realigning all the scans to the first scan. One subject with excessive head motion (more than $2 \mathrm{~mm}$ in any axis) was excluded from the analysis. Functional scans were then spatially normalized to Montreal Neurological Institute (MNI) space and spatially smoothed with a 9-mm full-width at half-maximum (FWHM) Gaussian filter to reduce noise and minimize the effects of normalization errors.

In this study, the focus of fMRI analyses was on data related to feedback processing. Task-related and learningrelated changes in brain activity have been reported elsewhere [14]. Statistical analyses were performed first at the subject level and then at the group level. Fixed effect analyses were performed at the subject level using a general linear model in SPM5. The onsets of outcome presentations were modeled as delta functions convolved with a canonical hemodynamic response function. The model fit was performed and contrast images were generated for each of the six event types: feedback positive 1 (FBP1) (hit trials in the SPF task in scan session 1), feedback positive 2 (FBP2) (hit trials in the SPF task in scan session 2), feedback negative 1 (FBN1) (miss trials in the SPF task in scan session 1), feedback negative 2 (FBN2) (miss trials in the SPF task in scan session 2), control 1 (Cont1) (control trials in scan session 1), control 2 (Cont2) (control trials in scan session 2). Subtraction images were created from the contrasts as follows. (FBP1 - Cont1) - (FBP2 - Cont2), for assessing learningrelated increases in brain activity related to positive feedback processing; (FBP2 - Cont2) - (FBP1 - Cont1), for assessing learning-related decreases in brain activity related to positive feedback processing; (FBN1 - Cont1) - (FBN2 - Cont2), for assessing learning-related increases in brain activity related to negative feedback processing; (FBN2 - Cont2) - (FBN1 Cont1), for assessing learning-related decreases in brain activity related to negative feedback processing. There were two reasons for subtracting brain responses to control events from responses to feedback events. First, to exclude the activity associated with response-generation portion of the task in each session. Second, to cancel out the learning-related changes in activity associated with response generation.

For assessing learning-related changes in brain activity associated with feedback processing, at the group-level random effect analyses, inter-subject maps were created by performing a one-sample t-test on each of the contrast images. The statistical threshold was set at $\mathrm{p}<0.001$ (cluster-level corrected, $\mathrm{p}<0.05)$. Finally, the resulting activation maps were constructed and superimposed onto stereotactically standardized T1-weighted MRI images.

\section{RESULTS}

\section{Behavioral Results}

Significant changes in performance between the two scan sessions were observed for SPF task $(t=3.74, p<.01)$. The 
mean $( \pm \mathrm{SD})$ success rate was $22.8 \%$ (2.8) in the first scan session (percentage of trials in which subjects achieved the target) and increased to $39.9 \%$ (4.2) in the second scan session. The mean number of positive/negative feedback events was $21 / 68$ in the first scan session and increased to $36 / 54$ in the second scan session. The number of positive feedback events was significantly higher in the second scan session than in the first session $(\mathrm{t}=4.6, \mathrm{p}<0.01)$.

Immediately after the second scan session, a short interview was conducted with the subjects. As a result of the interviews, we learned that none of the subjects had discovered the hidden rule and that describing the reason for their improvement verbally was difficult for them. Specifically, many of the subjects could not provide any description of their improvement in task performance. The statements that were made by other subjects about their strategies were not informative, e.g. "setting the initial number of workers based on the target value (e.g. 8 workers for 8000 tons of sugar output) and then adjusting it appropriately based on my experience in the practice session".

\section{Neuroimaging Results}

The coordinates of activations and their extent are listed in Table 1 and depicted in Fig. (2). The beta-estimates of activity (before and after training) which represent the percent signal change in the activated regions are also depicted in Fig. (2). Regarding positive feedback processing in the SPF task, the only brain region that showed significant learning-related decrease in activity was the right superior frontal gyrus (Fig. 2A). No significant learning-related increase in activity has been observed in response to positive feedback. In addition, the results showed a significant learning-related increase in activity in the left precuneus (Fig. 2B) and middle occipital gyrus (Fig. 2C) in response to negative feedback. In contrast to positive feedback processing, no significant learning-related decrease in activity has been observed in response to negative feedback.

\section{DISCUSSION}

In recent years, several neuroimaging studies investigated the neural correlates of positive and negative feedback processing during the course of learning $[2,4,10,11]$. In this study, a different perspective has been taken to investigate the interplay between feedback processing and learning. We examined the changes in neural substrates underlying outcome feedback processing during a goal-directed implicit learning paradigm. Comparison of brain activity associated with feedback processing before and after learning showed that learning the SPF task affects the neural substrates underlying positive and negative feedback processing. In response to positive feedback, a significant learning-related decrease in activity was found in the right SFG which has been previously reported to be involved in the exploratory phase of learning the SPF task [14]. On the other hand, significant learning-related increases in activity were found in the left precuneus and middle occipital gyrus in response to negative feedback. Learning-related change in the activity of left precuneus has also been observed in the same experiment [14]. These findings demonstrate the changing role of feedback during learning and suggest that learning-related changes in the activity of SFG and precuneus that have been previously reported in several implicit learning studies arise from changes in feedback processing after learning.

\section{Effects of Learning on Positive Feedback Processing}

We found a learning-related decrease in activation of the right SFG related to processing of positive feedback. A decrease in the activation of this brain region after practice in the second scan session has also been observed in our study of learning the SPF task [14]. Several studies reported decreasing activation of the right medial SFG with increasing performance and speculated that this brain region may be involved in performance evaluation [27] and evaluating internal states [28-30]. However, the exact contribution of this brain region in performance evaluation is not clear. The results of the present study suggest that decremental activation of the right SFG with rehearsal is specifically related to decreasing demand for positive feedback processing after learning. Rushworth and colleagues reviewed the functions of the medial frontal cortex and suggest that the superior frontal region, compared to other regions, is most important whenever actions sets are initiated by selecting superordinate sets of action-selections rules [31]. In the explanatory phase of learning the SPF task, correct responses signal the involvement of this brain region to select the latest applied stimulus-response-outcome association rules as the successful one. However, after learning, this process may be less required as this association is almost established.

Table 1. Learning-Related Changes in Brain Activity in Response to Positive and Negative Feedback in the SPF Task

\begin{tabular}{|c|c|c|c|c|}
\hline \multirow[t]{2}{*}{ Brain area } & \multicolumn{3}{|c|}{ Coordinates } & \multirow[t]{2}{*}{$t$-value } \\
\hline & $x$ & $y$ & $z$ & \\
\hline \multicolumn{5}{|c|}{ Learning-related decreases in response to positive feedback } \\
\hline R Superior frontal gyrus ${ }^{*}$ & 12 & 6 & 64 & 10.5 \\
\hline \multicolumn{5}{|c|}{ Learning-related increases in response to negative feedback } \\
\hline \multirow[t]{2}{*}{ L Precuneus* } & 14 & 74 & 36 & 7.05 \\
\hline & 28 & 74 & 34 & 6.53 \\
\hline \multirow[t]{2}{*}{ L Middle occipital gyrus } & 32 & 82 & 16 & 9.86 \\
\hline & -16 & -94 & 12 & 5.52 \\
\hline
\end{tabular}

Abbreviations: L, left; R, right. "Corrected for multiple comparison at cluster-level, p <0.05, extent of at least 10 voxels. 


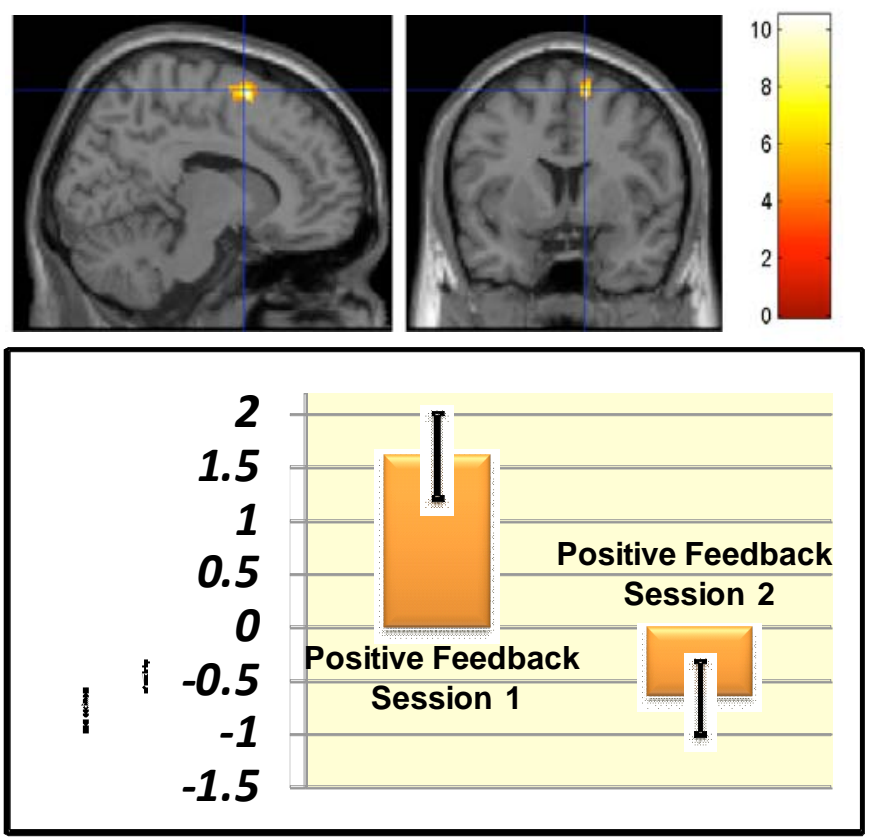

A

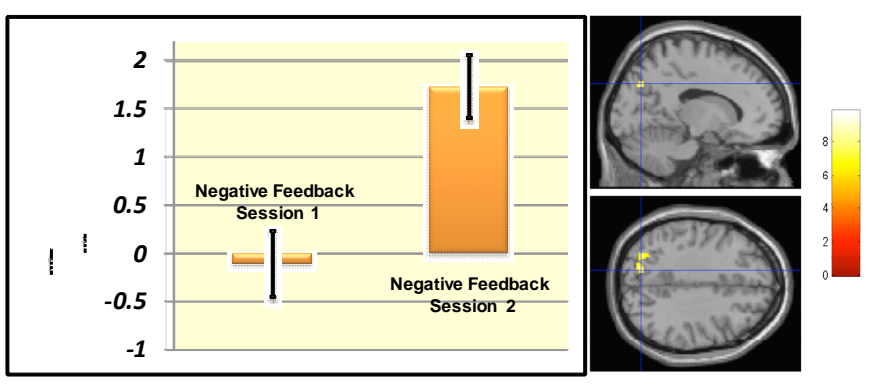

B

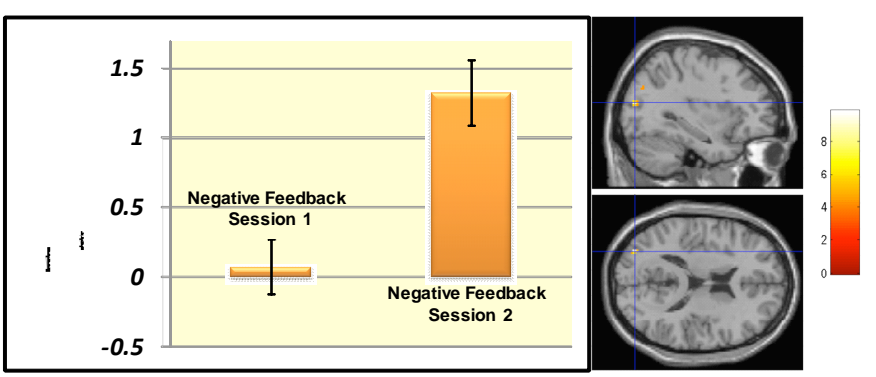

C

Fig. (2). (A) Decreases in activation of right SFG after training in response to positive feedback (top) and beta estimate of activity in this region before and after training (bottom). (B) Increases in activation of left precuneus after training in response to negative feedback (right) and beta estimate of activity in this region before and after training (left). (C) Increases in activation of middle occipital gyrus after training in response to negative feedback (right) and beta estimate of activity in this region before and after training (left).

\section{Effects of Learning on Negative Feedback Processing}

We found a learning-related increase in activation of the precuneus related to processing of negative feedback. The precuneus also exhibited increased activation with training on the SPF task in the same experiment [14]. The learningrelated increase in the activity of this brain region has been also found in our previous meta-analysis on 17 implicit learning studies [32]. Although learning-related increase in the activity of the precuneus has been observed in previous implicit learning studies [22, 33], the exact role of this brain region in implicit learning is still controversial. Previous neuroimaging studies on feedback-based learning speculated that this brain region is involved in attention-set shifting [34], sequence learning [35], supplying alternative strategies [36] and evaluative feedback processing [37]. While the observed precuneus activity following negative feedback might also reflect supplying an alternative strategy or shifting in attention-set, our results suggest that the observed learningrelated increase in the precuneus activity is associated with increasing response to negative feedback after training. The involvement of precuneus in negative feedback processing has been reported in a recent neuroimaging study on feedback-based learning [38]. They found that precuneus exhibited greater activation in response to negative than positive feedback. In line with this finding, Eliassen et al. [23] showed that precuneus exhibited greater activation for incorrectly than correctly performed trials in an associative learning task and suggest its involvement in error processing. Therefore, the precuneus activation in response to negative feedback after training might cause a stronger attention focus on the outcome situation (visual perception and processing of the outcome) [39]; An idea that also explains the observed activation in the visual attention area, i.e. the middle occipi- tal gyrus, in response to negative feedback after training. Recent evidence indicates functional organization within the precuneus along an anterior/mid/posterior axis; the anterior part involves in sensorimotor processes, the central part involves in cognitive/associative processes, and the posterior part involves in visual processes [40]. Although it is difficult to address the functional subregion specialization based on our event-related fMRI data on $1.5 \mathrm{~T}$ scanner, the mapping results show that the observed activity lies in the central cognitive/associative region. This region of the precuneus has strong connectivity with the dorsolateral prefrontal and inferior parietal cortices [41] implicated in higher order executive processing; supporting our idea that observed learning-related increase in the central precunal activation in response to negative feedback might reflect signaling a stronger attention focus on the outcome situation.

The other interesting observation in the present study was that we neither find any significant learning-related increase in brain activity in response to positive feedback nor significant learning-related decrease in response to negative feedback. In other words, whereas the activation following negative feedback increased with learning, activation following positive feedback decreased. It implies that in the exploratory phase of learning, compared to late stage of learning, subjects more focused on the information from positive feedback to improve their performance. While in the late stage of learning, negative feedback weighs more for subjects to keep their performance compared to early stage of learning. These results suggest the important role of positive and negative feedback in early and late stages of goaldirected implicit learning, respectively.

In summary, the SPF task, as a goal-directed implicit learning paradigm, in conjunction with event-related fMRI 
has been used to investigate the learning-related changes in neural substrates underlying positive and negative feedback processing. The results suggest the involvement of the right SFG in positive feedback processing in exploratory phase of learning that explains learning-related decrease in activity of this region observed previously in implicit learning studies. The results also suggest that the observed learning-related increase in the precuneus activity in previous implicit learning studies arises from the specific role of this brain region in negative feedback processing after learning. Besides clarifying the role of the right SFG and precuneus in implicit learning, the results revealed the important role of positive and negative feedback in early and late stages of learning, respectively.

\section{REFERENCES}

[1] Thorndike EL. Animal intelligence: experimental studies. New York: MacMillan 2000.

[2] Aron AR, Shohamy D, Clark J, Myers C, Gluck MA, Poldrack RA. Human midbrain sensitivity to cognitive feedback and uncertainty during classification learning. J Neurophysiol 2004; 92: 1144 -52.

[3] Elliott R, Newman JL, Longe OA, Deakin JF. Differential response patterns in the striatum and orbitofrontal cortex to financial reward in humans: a parametric functional magnetic resonance imaging study. J Neurosci 2003; 23: 303-7.

[4] Evers EA, Cools R, Clark L, et al. Serotonergic modulation of prefrontal cortex during negative feedback in probabilistic reversal learning. Neuropsychopharmacol 2005; 30: 1138-47.

[5] Knutson B, Westdorp A, Kaiser E, Hommer D. FMRI visualization of brain activity during a monetary incentive delay task. NeuroImage 2000; 12: 20-7.

[6] Marco-Pallares J, Müller SV, Munte TF. Learning by doing: an fMRI study of feedback-related brain activations. Neuroreport 2007; 18: 1423-6.

[7] Nieuwenhuis S, Slagter H, Alting Von Geusau N, Heslenfeld DJ, Holroyd CB. Knowing good from bad: Differential activation of human cortical areas by positive and negative outcomes. Eur J Neurosci 2005; 21: 3161-8.

[8] Ullsperger M, Von Cramon DY. Error monitoring using external feedback: specific roles of the habenular complex, the reward system, and the cingulate motor area revealed by functional magnetic resonance imaging. J Neurosci 2003; 23: 4308-14.

[9] Klein TA, Neumann J, Reuter M, Hennig J, Von Cramon DY, Ullsperger M. Genetically determined differences in learning from errors. Science 2007; 318: 1642-5.

[10] Seger CA, Cincotta CM. The roles of the caudate nucleus in human classification learning. J Neurosci 2005; 25: 2941-51.

[11] Shohamy D, Myers CE, Grossman S, Sage J, Gluck MA, Poldrack RA. Cortico-striatal contributions to feedback-based learning: converging data from neuroimaging and neuropsychology. Brain 2004; 127: 851-9.

[12] Sailer U, Robinson S, Fischmeister FP, Moser E, Kryspin-Exner I, Bauer $\mathrm{H}$. Imaging the changing role of feedback during learning in decision-making. NeuroImage 2007; 37: 1474-86.

[13] Valentin VV, Dickinson A, O'Doherty JP. Determining the neural substrates of goal-directed learning in the human brain. J Neurosci 2007; 27: 4019-26.

[14] Rostami M, Hosseini SMH, Takahashi M, Sugiura M, Kawashima R. Neural bases of goal-directed implicit learning. NeuroImage 2009a; 48: 303-10.

[15] Berry DC, Broadbent DE. On the relationship between task performance and associated verbalizable knowledge. Quart J of Exp Psychol 1984; 36: 209-31.

[16] Berry DC, Broadbent DE. The combination of explicit and implicit learning processes in task control. Psycholo Res 1987; 49: 7-15.

[17] Berry DC, Broadbent DE. Interactive tasks and the implicitexplicit distinction. Brit J Psychol 1988; 79: 251-72.
[18] Dalley JW, Cardinal RN, Robbins TW. Prefrontal executive and cognitive functions in rodents: neural and neurochemical substrates. Neurosci Biobehav Rev 2004; 28: 771-84.

[19] Killcross S, Coutureau E. Coordination of actions and habits in the medial prefrontal cortex of rats. Cerebral Cortex 2003; 13: 400-8.

[20] Ostlund SB, Balleine BW. Lesions of medial prefrontal cortex disrupt the acquisition but not the expression of goal-directed learning. J Neurosci 2005; 25: 7763-70.

[21] Chein JM, Schneider W. Neuroimaging studies of practice-related change: fMRI and meta-analytic evidence of a domain-general control network for learning. Cogn Brain Res 2005; 25: 607-23.

[22] Poldrack RA, Desmond JE, Glover GH, Gabrieli JDE. The neural basis of visual skill learning: an fMRI study of mirror-reading. Cereb Cortex 1998; 8: 1-10.

[23] Eliassen JC, Souza T, Sanes JN. Experience-dependent activation in human brain during visual-motor associative learning. J Neurosci 2003a; 23: 10540-7.

[24] Oldfield RC. The assessment and analysis of handedness: the Edinburgh Inventory. Neuropsychologia 1971; 9: 97-113.

[25] Friston KJ, Ashburner L, Frith CD, Poline JB, Hether JD, Frackowiak RS. Spatial registration and normalization of images. Hum Brain Mapp 1995a; 3: 165-89.

[26] Friston KJ, Holmes AP, Worsley KJ, Poline JB, Frith CD, Frackowiak RSJ. Statistical parametric maps in functional imaging: a general linear approach. Human Brain Mapping 1995b; 2: 189-210.

[27] Eliassen JC, Johnson MB, Sanes JN. Experience dependent modulation of stimulus- and feedback-related hemodynamic responses during arbitrary association learning. NeuroImage 2003b; 19: Supplement 1 .

[28] Castelli F, Happe F, Frith U, Frith C. Movement and mind: a functional imaging study of perception and interpretation of complex intentional movement patterns. NeuroImage 2000; 12: 314-25.

[29] Gusnard DA, Akbudak E, Shulman GL, Raichle ME. Medial prefrontal cortex and self-referential mental activity: relation to a default mode of brain function. PNAS 2001; 98: 4259-64.

[30] Zysset S, Huber O, Ferstl E, Von Cramon DY. The anterior frontomedian cortex and evaluative judgment: an fMRI study. NeuroImage 2000; 15: 983-91.

[31] Rushworth MFS, Walton ME, Kennerley SW, Bannerman DM. Action sets and decisions in the medial frontal cortex. Trends Cogn Sci 2004; 8: 410-17.

[32] Rostami M, Hosseini SMH, Takahashi M, Kawashima R. Neural mechanisms of skill acquisition: a meta-analysis. NeuroImage 2009b; 47: s39-s41.

[33] Aizenstein HJ, Stenger VA, Cochran J, Clark K, Johnson M, Nebes $\mathrm{RD}$, Carter CS. Regional brain activation during concurrent implicit and explicit sequence learning. Cereb Cortex 2004; 14: 199208.

[34] Nagahama Y, Okada T, Katsumi Y, Hayashi T, et al. Transient neural activity in the medial superior frontal gyrus and precuneus time locked-activity with attention shift between object features. NeuroImage 1999; 10: 193-9.

[35] Sakai K, Hikosaka O, Miyauchi S, Takino R, Sasaki Y, Pütz B. Transition of brain activation from frontal to parietal areas in visuo: motor sequence learning. J Neurosci 1998; 18: 1827-40.

[36] Paulus MP, Hozack N, Zauscher B, McDowell JE, Frank L, Brown GG, Braff DL. Prefrontal, parietal, and temporal cortex networks underlie decision-making in the presence of uncertainty. NeuroImage 2001; 13: 91-100.

[37] Xiaohong P, Hu Y, Li L, Li J. Evaluative-feedback stimuli selectively activate the self-related brain area: An fMRI study. Neurosci Lett 2009; 465: 90-4.

[38] Zanoliea K, Tengd S, Donohuee SE, et al. Switching between colors and shapes on the basis of positive and negative feedback: an fMRI and EEG study on feedback-based learning. Cortex 2008; 44: 537-47.

[39] Koeneke S, Pedroni AF, Dieckmann A, Bosch V, Jancke L. Individual preferences modulate incentive values: evidence from functional MRI. Behavior and Brain Func 2008; 4: 55. 
[40] Margulies DS, Vincent JL, Kelly C, et al. Precuneus shares intrinsic functional architecture in humans and monkeys. PNAS 2009; 106: 20069-74.
[41] Morecraft RJ, Cipolloni PB, Stilwell-Morecraft KS, Gedney MT, Pandya DN. Cytoarchitecture and cortical connections of the posterior cingulate and adjacent somatosensory fields in the rhesus monkey. J Comp Neurol 2004; 469: 37-69.

(C) Rostami et al.; Licensee Bentham Open.

This is an open access article licensed under the terms of the Creative Commons Attribution Non-Commercial License (http://creativecommons.org/licenses/by-nc/3.0/) which permits unrestricted, non-commercial use, distribution and reproduction in any medium, provided the work is properly cited. 